\title{
Spatial models of imagery for remembered scenes are more likely to advance (neuro)science than symbolic ones
}

\section{Neil Burgess}

Institute of Cognitive Neuroscience and Department of Anatomy, University College London, London, WC1E 6BT, United Kingdom.

n.burgess@ucl.ac.uk http://www.icn.ucl.ac.uk/members/Burge12/

Abstract: Hemispatial neglect in imagery implies a spatially organised representation. Reaction times in memory for arrays of locations from shifted viewpoints indicate processes analogous to actual bodily movement through space. Behavioral data indicate a privileged role for this process in memory. A proposed spatial mechanism makes contact with direct recordings of the representations of location and orientation in the mammalian brain.

Pylyshyn's target article omits some of the evidence for the spatial organisation of visual imagery to be found in studies of memory for spatial scenes or arrays of objects. While not conclusive, this evidence may be instructive in escaping some of the logical caveats raised by Pylyshyn, and extending the discussion of the functional space in which retrieval products from memory are processed. Although other caveats will be found regarding these data, interpreting them in terms of their mapping onto space and our phys- 
ical movements within it will take us closer to understanding the relevant neural mechanisms. Thus, since science advances by a process in which one flawed but partially explanatory theory replaces another flawed but slightly-less explanatory theory, the spatial interpretation appeals to me as a neuroscientist. The evidence discussed here concerns (1) the spatial organisation of hemi-spatial neglect in imagery; (2) reaction time and performance data in memory for spatial locations; (3) the neuronal mechanisms suggested by single unit recordings in animals.

In patients with hemi-spatial neglect, damage to the internal image or to the means of accessing it occurs preferentially to the side contra-lateral to the lesion. How could this be unless the internal image itself were spatially organised? Pylyshyn (sect. 7.1) discusses Farah et al.'s (1992) patient who shows tunnel vision and also similar tunnel imagery. He argues that this patient has simply learned to simulate her impaired visual perception in imagery, that is, that this may not relate to the "cognitive architecture" of imagery. Can this objection be applied to hemi-spatial neglect in imagery? The majority of patients showing hemispatial neglect in imagery also show a similar perceptual neglect (Bisiach et al. 1979; 1981), indicating significant overlap between the architecture of the two systems. However, the caveat that imagery might imitate perception is ruled out by the (albeit much rarer) case of patients showing relatively pure imaginal neglect (Beschin et al. 1997; Guariglia et al. 1993), and even imaginal neglect on one side and perceptual neglect on the other (Beschin et al. 2000).

The second piece of evidence concerns memory for the locations of objects in an array following a change in viewpoint. In these experiments, reaction times show a linear dependence on the size of the change in the subject's location or orientation between presentation and retrieval (Diwadkar \& McNamara 1997). Related imagery experiments require the subject, previously shown an array of locations, to point in the direction a location would have following a (imagined) rotation or translation of the subject. These experiments show a similar dependence of reaction time on the size of the rotation or translation between the subject's current position and the position from which they should imagine pointing (Easton \& Sholl 1995). These tasks probably differ from those involving single objects (e.g., Shepherd \& Metzler 1971) in being solved by imagined movement of viewpoint as opposed to an equivalent imagined movement of the array (for which RTs and performance are worse). Only when a single object need be considered can imagined rotation of the array produce performance approaching that for imagined movement of viewpoint (Wraga et al. 2000).

The same caveats apply to the interpretation of viewpoint manipulation data that Pylyshyn raises against mental rotation of single objects. However, in this case, there is independent evidence that our "cognitive architecture" is specifically adapted to accommodate the effects of physical movement through the environment compared to an equivalent movement of the array (Simons \& Wang 1998; Wang \& Simons 1999). In these experiments, subjects' recognition memory for an array of objects on a circular table top is better after the subject had moved around the table to a new viewpoint than after an equivalent rotation of the table top. Since this effect is also observed in the dark (using phosphorescent objects) and in purely visual virtual reality (Christou \& Bulthoff 1999), the facilitation appears to apply to any processes corresponding to movement of viewpoint within the subject's mental model of the world.

What are the neural bases of these processes? A patient with focal damage to both hippocampi is specifically impaired at shifted view recognition of two or more object-locations compared to fixed-view recognition, or shifted view recognition of a single object-location (King et al. 2002). The neural bases of self-location and orientation have been well examined in the rat. "Place cells" in the hippocampus encode the animal's current location in the environment (O’Keefe 1976; Wilson \& McNaughton 1993) while "head-direction cells" nearby in the presubiculum (also mammillary bodies and anterior thalamus) encode its current orientation
(Taube et al. 1990). Additionally, cells in the connected area 7a of monkey parietal cortex represent stimulus locations in frames of reference relative to eye, head, and trunk, and allow translation between these frames and the environmental frame (Andersen et al. 1985; Pouget \& Sejnowski 1999; Snyder et al. 1998). Viewpointdependent retrieval of remembered places can be modelled as an interaction between the parietal, place and head-direction systems, possibly accounting for their involvement in episodic memory (Burgess et al. 2001). Interestingly, current models of the place and head-direction systems see each representation as a "continuous attractor" (Zhang 1996), in which the represented location or direction can shift under internal dynamics, but at a fixed speed (determined by the effective asymmetry of the connections between cells). This mechanism, applied to the viewpoint-dependent retrieval model, could explain the reaction time data, providing an explanatory model linking cells to spatial memory and imagery. Symbolic accounts seem less well formed to address these types of data. 